DOI: 10.12731/2070-7568-2020-4-281-295

УДК 366.1

\title{
АГЕНТ-ОРИЕНТИРОВАННОЕ МОДЕЛИРОВАНИЕ ПОВЕДЕНИЯ ПОТРЕБИТЕЛЕЙ НА РЫНКЕ МОЛОЧНОЙ ПРОДУКЦИИ
}

Отмахова Ю.С., Ибрагимов Н.М.

Проблемы разнонаправленных интересов участников продовольственного рынка приводят к различным трансформачиям потребительского спроса и актуализируют необходимость исследования и определения различных стратегий поведения потребителей на рынках сочиально-значимой пищевой продукции. В работе представлен авторский подход к построению агентно-ориентированной модели поведения потребителей на рынке молочной продукции в условиях информационной асимметрии и ограниченной рациональности действий агентов. Результаты проведенных вычислимых экспериментов позволили продемонстрировать стратегии поведения потребителей с изменением таких факторов как пищевые предпочтения, уровень иен, частота совершения покупки, степень приверженности к принципам здорового питания, лояльность к бренду, степень восприимчивости рекламной информации.

Цель - разработать агент-ориентированную модель поведения потребителей молочной продукиии с учетом ограниченной рациональности действий агентов.

Метод и методология проведения работы: агент-ориентированный подход кмоделированию рынка и модели потребительского поведения.

Результаты. Результаты исследования представляют теоретическую основу для дальнейшего развития теории потребительского поведения и имитационного моделирования.

Область применения результатов. Результаты исследования могут быть использованы при определении инструментов государственного управления на рынке продуктов питания для снижения 
уровня асимметрии информации по потребительским характеристикам продуктов питания.

Ключевые слова: агент-ориентированное моделирование; пищзевые предпочтения; выбор пищевого продукта; имитационное моделирование; теория рыночного спроса; теория потребительского поведения.

\section{AGENT-BASED MODELING OF CONSUMER BEHAVIOR ON DAIRY MARKET}

\section{Otmakhova Yu.S., Ibragimov N.M.}

The problems of divergent interests of food market participants lead to various transformations of consumer demand and actualize the need to research and determine various strategies for consumer behavior in the markets of socially significant food products. The paper presents the author's approach to building an agent-based model of consumer behavior in the dairy market in terms of information asymmetry and limited rationality of agents. The results of the conducted computable experiments allowed us to demonstrate the strategies of consumer behavior with changes in such factors as food preferences, price level, frequency of purchase, the degree of adherence to the principles of healthy eating, brand loyalty, and the degree of susceptibility of advertising information.

Purpose: develop agent-based model of behavior of the dairy consumers using the limited rationality of agents 'actions.

Methodology: agent-based approach to market modeling and consumer behavior models.

Results. The obtained results represent the theoretical basis for development of the theory of consumer behavior and simulation modeling.

Practical implications. The results could be used to determine the tools of state regulation in the food market to reduce the level of asymmetry of information on consumer characteristics of food.

Keywords: agent-based modeling; food preference; food choice; simulation modeling; market demand; consumer behavior. 
В современных условиях при постоянно возрастающем предложении на рынке продуктов питания выбор потребителя значительно усложняется из-за значительного влияния информационной асимметрии, связанной с потребительскими характеристиками продуктов. Трансформационные изменения технологий производства продуктов питания носят глобальный характер и непосредственно отражаются на составе и качественных характеристиках продукта. Смена потребительских предпочтений и паттернов пищевого поведения вызывает изменения потребительского спроса на продукты питания и актуализирует необходимость разработки новых подходов и инструментария для моделирования и прогнозирования поведения потребителей на рынке пищевой продукции. Проблемы разнонаправленных интересов участников продовольственного рынка приводят к различным трансформациям потребительского спроса и актуализируют необходимость исследования и определение различных стратегий поведения потребителей на рынках социально-значимой пищевой продукции, в том числе на рынке молочных продуктов.

\section{Обзор подходов и инструментария}

для моделирования и прогнозирования поведения потребителей на рынке пищевой продукции

Потребительское поведение является междисциплинарной областью исследования, отечественные и зарубежные публикации по данной тематике представлены в таких областях знания как психология, социология, экономика, информатика, прикладная математика и др. Методы анализа и моделирования в области потребительского поведения весьма разнообразны и варьируются от традиционных количественных методов таких как статистический и регрессионный анализ до качественных методов в виде опросов, интервью и др.

Необходимо отметить, что в последнее время появляются новые исследования, в которых для оценки влияния на потребительское поведение значительного количества факторов, используются современные вычислительные методы, основанные на компьютер- 
ном моделировании, интеллектуальном анализе данных, анализе больших данных, нейронных сетях, машинное обучение $[13,17]$.

Ранее авторы данной статьи в работе [5] отмечали, что проблема информационной асимметрии находит свое выражение в том, что большинство производителей пищевой продукции ориентируются на высокодоходный сегмент пищевой продукции с низкой долей натурального сырья в ущерб качеству и пищевой ценности продуктов питания промышленного производства.

Рынок продовольственных продуктов отличается разнообразием покупательских предпочтений среди достаточно широкого круга потребителей Разнонаправленные интересы и сложные коммуникативные взаимодействия участников продовольственного рынка обуславливают существенные трудности для традиционного статистического и эконометрического анализа. В западной литературе для характеристики рынка пищевых продуктов в индивидуальной упаковке закрепилось такое понятие как рынок потребительских упакованных товаров (consumer packaged goods - CPG). Сравнительно недавно рынок CPG стал рассматривать в рамках анализа «сложных систем» $[14,15,16]$, в результате для его анализа и прогнозирования используются наиболее современные методологии “снизу вверх”, такие как агентное моделирование [11].

Методы моделирования, основанные на конструкциях, основанных на агентах, где составляющие системы (покупатели, компании и др.) рассматриваются как отдельные единицы моделирования (или агенты), способные следовать независимым правилам поведения, целях и стратегиям взаимодействия, становятся все более популярными $[8,9,10,18]$. Основная идея агентного подхода заключается в построении вычислительного комплекса, представляющего собой множество агентов с определенным набором свойств и правил поведения и взаимодействия [12]. Агент-ориентированный подход к моделированию универсален и удобен в силу своей наглядности, но при этом предъявляет высокие требования к вычислительным ресурсам [1].

В динамичной, конкурентной и сложной среде продовольственного рынка выбор покупателя зачастую зависит от его пищевых 
предпочтений, рациона питания, моделей пищевого поведения, уровня дохода, образования, а также внешних влияний, которые могут быть учтены при реализации модели с использованием агентных моделей.

Агропродовольственный рынок вызывает интерес исследователей в области агентного моделирования и в последние несколько лет постепенно расширяется использование подходов к моделированию на основе агентов в рамках оценки сельскохозяйственной и индустриальной политики [7]. Среди довольно немногочисленных работ по АОМ рынка продовольствия входит и статья авторов, посвященная моделированию мирового рынка ячменя [4]. В работе проведена серия вычислимых экспериментов в среде AnyLogic с изменением таких факторов, как уровень глобального спроса, величина пошлины, размер капитала компаний-экспортеров для определения стратегии поведения агентов-экспортеров.

В ряде публикаций отмечается, что современное товарное предложение на рынке молока характеризуется увеличением доли продукции ненадлежащего качества. Рядом исследователей отмечается тенденция перехода продуктов питания в разряд доверительных благ, что неизбежно сопровождается ростом информационной асимметрии и проблемой ухудшающегося отбора (снижение качества) [3]. Молочная продукция являются важной категорией в структуре питания населения России, что определяет значимость исследования комплекса взаимоотношений и противоречивых интересов участников рынка и требует формализации задачи с использованием современного инструментария имитационного моделирования.

\section{Методы и инструментарий исследования}

Агентные модели реализуются с помощью различных программных средах (к примеру, в Eclipse, MS Visual Studio и др.) [2]. В рамках исследования в качестве инструментария было использовано современное программное обеспечение для имитационного моделирования, разработанного российской компании «The AnyLogic Company». Разработанная в рамках данного исследования AOM 
рынка молочных продуктов была реализована в среде AnyLogic версия 8.7.1 University Edition (тестовый доступ).

В качестве объекта исследования был выбран рынок молочных упакованных продуктов (на примере сыра твердого). В процессе формирования модели агенты-покупатели формировалось в зависимости от выделенных типов покупателей, сформированных на основе анализа эмпирических данных, действующих с позиции ограниченной рациональностью выбора. Метод сбора эмпирических данных - анкетный опрос, представлен в работе авторов [6]. В работе на модельном примере на основе эмпирических данных были сформированы три основных типов агентов и сформированы их стратегий поведения.

В рамках предлагаемого подхода считаем, что влияние сложных факторов внешней среды на потребительский выбор и пищевые предпочтения может быть проанализировано через призму анализа потребительского поведения, тогда как вариации персональные характеристики потребителя (атрибуты) такие как возраст, пол, уровень образования, занятость, и др.) могут определять индикаторы потребительских паттернов поведения (пищевые предпочтения, частота употребления молочных продуктов, степень восприятия рекламной информации, степень приверженности к принципам здорового питания и др.). Под стратегиями потребительского поведения нами понимается принципы поведения покупателей, которые определяют индивидуальные особенности выбора и приобретения упакованного молочного продукта, а также потребительские предпочтения относительно вкуса, консистенции, бренда, стоимости и состава продукта.

\section{Характеристика модели}

При формировании модельного примера исходили из следующих положений и обозначений:

1) Агенты-потребители в рамках модельного примера постоянно проживают на территории одного города, включающего пять районов. Потребители могут приобрести продукт (сыр твердый) в 
любом районе города, их возможность перемещения определяется в модели через уровень изменения локации (relocation rate). Агенты-потребители могут изменять свое решение о покупке сыра в соответствии с выделенным типом стратегии в связи определенным уровнем восприятия рекламной информации и рекомендаций других потребителей (influence weight), уровень воздействия рекламных сообщений и степень лояльности к бренду (remaining influence). Агентам в модели определена ежедневная норма потребления сыра и необходимость покупки (daily need). У каждого потребителя есть свои пищевые предпочтения и свой вид сыра, который он предпочитает, однако под влиянием от изменения цены, рекламных акций, общения с другими агентами или с определенной долей случайности потребитель может изменить свои предпочтения.

2) Предполагалось, что изначально три компании-производители молочных продуктов (Red, Green, Blue) выходят на рынок с определенной ценой продукта (price), которая складывается из производственных затрат компании и варьируется в диапазоне средних цен на сыр твердый. Компании может продавать сыр в любом районе городе. Размер бюджета на продвижение и рекламу продукта агентов-производителей позволяет проводить акцию по продвижению на территорию всего города (global promotion) или на территории одного района города (local promotion) с определенной длительностью (contact promotion).

Формальное описание предлагаемой агент-ориентированной модели включает характеристику агентов-компаний производителей, параметры для потребления и параметры взаимодействия для агентов-потребителей.

В рамках модели были определены следующие функции агентов: функция перемещения агента-потребителя в рамках районов города FindLocation; функция выбора случайного выбора района для агента-потребителя RandomDistrict; функция определения границ области перемещения агентов (внутри районов или района) DistrictBounds; функция определения вида продукта с наибольшим предпочтением MaxPreference; функция проведение ме- 
роприятий по продвижению продукта на территории всех районов города (globalPromotion). В модели в качестве единицы модельного времени выбраны дни, количество агентов составило 2000, целевой функцией модели является максимизация прибыли агентов-производителей. Программная реализация модели позволяет зафиксировать перемещение агентов-потребителей как в отдельном районе городе, так позволить агентам перемещаться внутри всех районов. Модель позволяет задавать цену на товар, ежедневную потребность потребителей, уровень взаимодействия с другими агентами, уровень восприятия рекламной информации

\section{Результаты исследования}

В рамках исследования была проведена серия вычислимых экспериментов по агент-ориентированной модели поведения потребителей молочной продукции с учетом ограниченной рациональности действий агентов, реализованной в среде AnyLogic. Графическая среда модели позволила продемонстрировать динамику изменения доли рынка компаний-производителей (Market share) в виде меняющейся круговой диаграммы, динамику изменения общего дохода компании в виде столбиковой диаграммы, динамику продаж сыра в виде временной диаграммы с накоплением (Sales Dynamic), динамику еженедельных доходов компаний (Weekly Revenue) от продаж сыра на рынке. Далее приведены результаты проведенных вычислительных экспериментов на модельном примере при фиксированной цене на сыр для типа потребителя C1 с высоким уровнем восприимчивости к рекламной информации и мнению других агентов, имеющий возможность перемещаться между районами города в процессе выбора продукта. Агенты-производители воздействуют на потребителей посредством применения рекламных акций во всех районах города длительностью 30 дней, а также специальных акций внутри районов длительностью 45 дней (рис. 1).

Результаты проведенных вычислимых экспериментов позволили продемонстрировать стратегии поведения потребителей для различных типов потребителей $(\mathrm{C} 1, \mathrm{C} 2, \mathrm{C} 3)$ с изменением таких факторов 
как пищевые предпочтения, уровень цен, частота совершения покупки, степень приверженности к принципам здорового питания, лояльность к бренду, степень восприимчивости рекламной информации.
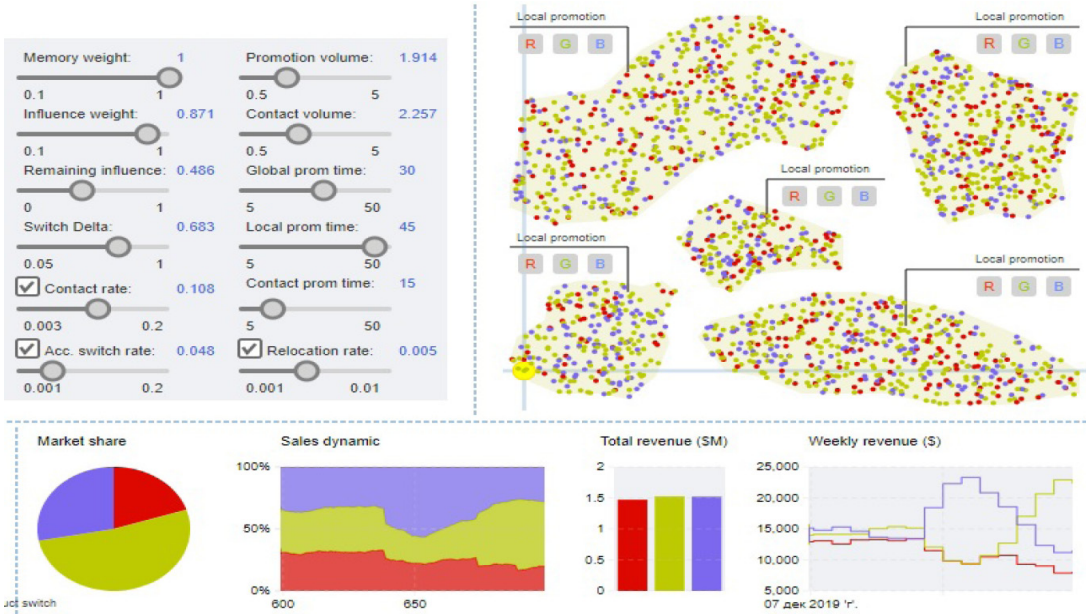

Рис. 1. Фрагмент результатов вычислительных экспериментов по агент-ориентированной модели.

Степень восприимчивости к рекламной информации и мнению других увеличивалась при увеличении числа контактов агентов и частотой перемещения агентов между районами. Увеличение продолжительности акций по продвижению товара на территории городе более 30 дней не оказывало существенное влияние на степень приверженности к рекламируемым продуктам компании и повышению лояльности.

\section{Заключение}

В современных условиях потребитель на рынке молочной продукции действует в условиях неопределенности относительно качества и натуральности исходного сырья, поэтому информационная асимметрия оказывает серьезное влияние на процесс принятия решения о выборе товара, а формализованное представление на модельном уровне может предоставить возможность оценки факторов, 
оказывающих влияния на потребительский выбор и определение мер государственного управления по нивелированию асимметрии на рынке молочной продукции. В условиях разнонаправленных интересов и сложных коммуникативных взаимодействий участников продовольственного рынка, широкого разнообразия потребительских предпочтений возникают существенные трудности для традиционного статистического и эконометрического анализа.

В рамках исследования была построена агентно-ориентированная модель поведения потребителей на рынке молочной продукции, на которой была проведена серия вычислимых экспериментов в среде AnyLogic с изменением таких факторов как пищевые предпочтения, уровень цен, частота совершения покупки, степень приверженности к принципам здорового питания, лояльность к бренду, степень восприимчивости рекламной информации.

Полученные результаты могут быть использованы при определении инструментов государственного управления на рынке продуктов питания для снижения уровня асимметрии информации по потребительским характеристикам продуктов питания. Разработанная модель с использованием агент-ориентированного подхода может стать полезным инструментом для руководства маркетинговыми стратегиями компаний и для понимания закономерностей конкуренции.

Финансирование. Статья подготовлена по плану НИР ИЭОПП CO РАН по проекту XI.171.1.1 «Разработка, апробация и применение в теоретических и прикладных исследованиях программно-методических комплексов и информационных систем анализа и прогнозирования социально-экономических процессов» № AAAA-A17-117022250129-2.

Funding. The publication is prepared within the project No. XI.171.1.1 "Development of program complexes and information systems for analysis and forecast of socio-economic process, their testing and implementation in theoretical and applied research", No. AAAA-A17-117022250129-2 according to the research plan of the IEIE SB RAS 


\section{Список литературы}

1. Бахтизин А.Р. Агент-ориентированные модели экономики. М.: Экономика, 2008. 279 с.

2. Макаров В.Л., Бахтизин А.Р., Сушко Е.Д., Васенин В.А., Борисов В.А., Роганов В.А. Агент-ориентированные модели: мировой опыт и технические возможности реализации на суперкомпьютерах // Вестник РАН. 2016. № 3. С. 252-262.

3. Малкина М.Ю. Институциональные основы снижения качества товаров и услуг в условиях рыночной экономики (ответ И.В. Розмаинскому) // Journal of Institutional Studies. 2014. Т. 6. № 4. С. 77-97.

4. Отмахова Ю.С., Ибрагимов Н.М. Возможности моделирования мирового рынка агропродовольственных товаров с использованием агенториентированного подхода // Мир экономики и управления. 2019. Т. 19, № 4. C. 104-113. DOI: 10.25205/2542-0429-2019-19-4-104-113

5. Усенко Н.И., Отмахова Ю.С., Оловянишников А.Г. Проблемы асимметрии корпоративных и общественных интересов на рынке продовольственных товаров // Экономические и социальные перемены: факты, тенденции, прогноз. 2014. № 3 (33). C. 124-139. DOI: 10.15838/ esc/2014.3.33.10.

6. Усенко Н.И., Яковлева Л.А., Отмахова Ю.С. Информационная асимметрия и особенности потребительского поведения на рынке молочной продукции // Техника и технология пищевых производств. 2016. T. 41. № 2. С. 156-163.

7. Abdou M., Hamill L., Gilbert N. Designing and building an agent-based model // Agent-Based Models of Geographical Systems. Springer, 2012, p. 141-165. DOI: 10.1007/978-90-481-8927-4

8. Ahrweiler, P. Agent-based simulation for science, technology, and innovation policy // Scientometrics 110, 391-415 (2017). DOI: 10.1007/ s11192-016-2105-0

9. Balke, T., and Gilbert, N. (2014). How do agents make decisions? A survey // Journal of Artificial Societies and Social Simulation, 17 (4), 13. DOI: $10.18564 /$ jasss. 2687

10. Baptista M.L., Martinho C., Lima F., Santos P.A., Prendinger H. (2014) A Business Simulation with an Agent-Based Deliberative Model of 
Consumer Behaviour. In: De Gloria A. (eds) Games and Learning Alliance. GALA 2013. Lecture Notes in Computer Science, vol 8605. Springer, Cham. https://doi.org/10.1007/978-3-319-12157-4_17

11. Gilbert, N., W. Jager, G. Deffuant, and I. Adjali (2007). Complexities in markets: Introduction to the special issue // Journal of Business Research 60(8), 813-815.

12. Hamill L., Gilbert N. Agent-Based Modelling in Economics. UK. Wiley 2015, 246 p.

13. B. J. Jacobs, B. Donkers, and D. Fok. Model-Based Purchase Predictions for Large Assortments // Marketing Science, 35(3):389-404, May 2016. DOI: $10.1287 / \mathrm{mksc} .2016 .0985$.

14. Rand, W. and Rust, R.T. Agent based modeling in marketing: Guidelines for rigor// International Journal for Research in Marketing 2011, 28(3), 181-193.

15. Sengupta, A. and Glavin, S.E. Volatility in a consumer packaged goods market: A simulation based study // Advances in Complex Systems 2010, 13(4), 579-605.

16. Sengupta, A. and Glavin, S.E. Predicting volatile consumer markets using multi-agent methods: Theory and validation. In Alexandrova-Kabadjova, B., Martinez-Jaramillo, S., Garcia-Almanza, A.L. and Tsang E. (Ed.) // Simulation in Computational Finance and Economics: Tools and Emerging Application. 2012. pp. 339-358. IGI Global.

17. M. Wan, D. Wang, M. Goldman, M. Taddy, J. Rao, J. Liu, D. Lymberopoulos, and J. Mcauley. Modeling Consumer Preferences and Price Sensitivities from Large-Scale Grocery Shopping Transaction Logs. Www, 2017. DOI: $10.1145 / 3038912.3052568$.

18. Weiping Wang, Saini Yang, Fuyu Hu, Zhangang Han, Carlo Jaeger. An agent-based modeling for housing prices with bounded rationality // IOP Conf. Series: Journal of Physics: Conf. Series 1113. 2018. №012014 DOI: $10.1088 / 1742-6596 / 1113 / 1 / 012014$

\section{References}

1. Bakhtizin A. R. Agent-orientirovannye modeli ekonomiki [Agent-oriented models of the economy]. Moscow, Ekonomika. 2008, 279 p. (in Russ.)

2. Makarov V.L., Bakhtizin A.R., Sushko E.D., Vasenin V.A., Borisov V.A., Roganov V.A. Supercomputer technologies in social sciences: Agent-ori- 
ented demographic models. Herald of the Russian Academy of Sciences, 2016, vol. 86, no. 3, p. 248-257.

3. Malkina M.Yu. Institutsional'nye osnovy snizheniya kachestva tovarov i uslug v usloviyakh rynochnoy ekonomiki (otvet I.V. Rozmainskomu) [Institutional frameworks of the reducing quality of goods and services in the market economy (the answer to I. V. Rozmainsky)]. Journal of Institutional Studies, 2014, vol. 6, no. 4, pp. 77-97.

4. Otmakhova Yu. S., Ibragimov N. M. Agent-Based Modeling of Global Agro-Food Market. World of Economics and Management. 2019. vol. 19, no. 4. P. 104-113. DOI: 10.25205/2542-0429-2019-19-4-104-113.

5. Usenko N.I., Otmakhova Y.S., Olovyanishnikov A.G. The issues of asymmetry of corporate and public interests in the food market. Economic and Social Changes: Facts, Trends, Forecast, 2014, no. 3 (33), pp. 124-139. DOI: $10.15838 / \mathrm{esc} / 2014.3 .33 .10$

6. Usenko N.I., Yakovleva L.M., Otmakhova Yu.S. Information asymmetry and consumer behavior in the market of dairy products. Food Processing: Techniques and Technology, 2016, vol. 41, no. 2, pp. 156-163 (in Russ.). Abdou M., Hamill L., Gilbert N. Designing and building an agent-based model. In: Agent-Based Models of Geographical Systems. Springer, 2012, pp. 141-165. DOI: 10.1007/978-90-481-8927-4

7. Abdou M., Hamill L., Gilbert N. Designing and building an agent-based model. Agent-Based Models of Geographical Systems. Springer, 2012, p. 141-165. DOI: 10.1007/978-90-481-8927-4

8. Ahrweiler, P. Agent-based simulation for science, technology, and innovation policy. Scientometrics 2017, 110, pp. 391-415. DOI: 10.1007/ s11192-016-2105-0

9. Balke, T., and Gilbert, N. How do agents make decisions? A survey. Journal of Artificial Societies and Social Simulation, 2014, 17 (4), 13. DOI: $10.18564 /$ jasss.2687

10. Baptista M.L., Martinho C., Lima F., Santos P.A., Prendinger H. (2014) A Business Simulation with an Agent-Based Deliberative Model of Consumer Behaviour. In: De Gloria A. (eds) Games and Learning Alliance. GALA 2013. Lecture Notes in Computer Science, vol 8605. Springer, Cham. https://doi.org/10.1007/978-3-319-12157-4_17 
11. Gilbert, N., W. Jager, G. Deffuant, and I. Adjali. Complexities in markets: Introduction to the special issue. Journal of Business Research 2007, 60(8), pp. 813-815.

12. Hamill L., Gilbert N. Agent-Based Modelling in Economics. UK. Wiley 2015, 246 p.

13. B. J. Jacobs, B. Donkers, and D. Fok. Model-Based Purchase Predictions for Large Assortments. Marketing Science, 35(3): pp. 389-404, May 2016. DOI: $10.1287 / \mathrm{mksc} .2016 .0985$.

14. Rand, W. and Rust, R.T. Agent based modeling in marketing: Guidelines for rigor. International Journal for Research in Marketing 2011, 28(3), 181-193.

15. Sengupta, A. and Glavin, S.E. Volatility in a consumer packaged goods market: A simulation based study. Advances in Complex Systems 2010, 13(4), 579-605.

16. Sengupta, A. and Glavin, S.E. Predicting volatile consumer markets using multi-agent methods: Theory and validation. In Alexandrova-Kabadjova, B., Martinez-Jaramillo, S., Garcia-Almanza, A.L. and Tsang E. (Ed.), Simulation in Computational Finance and Economics: Tools and Emerging Application. 2012. pp. 339-358. IGI Global.

17. M. Wan, D. Wang, M. Goldman, M. Taddy, J. Rao, J. Liu, D. Lymberopoulos, and J. Mcauley. Modeling Consumer Preferences and Price Sensitivities from Large-Scale Grocery Shopping Transaction Logs. Www, 2017. DOI: $10.1145 / 3038912.3052568$.

18. Weiping Wang, Saini Yang, Fuyu Hu, Zhangang Han, Carlo Jaeger. An agent-based modeling for housing prices with bounded rationality. IOP Conf. Series: Journal of Physics: Conf. Series 1113. 2018. №012014 DOI: $10.1088 / 1742-6596 / 1113 / 1 / 012014$

\section{ДАННЫЕ ОБ АВТОРАХ}

Отмахова Юлия Сергеевна, кандидат экономических наук, старший научный сотрудник лаборатории моделирования и анализа экономических процессов; заведующий лабораторией «Исследовательский центр продовольственной безопасности» Институт экономики и организачии промьииленного производства СО РАН; Новосибирский государственный университет пр. Акад. Лаврентьева, 17, г. Новосибирск, 630090, Российская 
Федерация; ул. Пирогова, 1, г. Новосибирск, 630090, Российская Федерачия

Ибрагимов Наимджон Мулабоевич, кандидат экономических наук, доцент, старший научный сотрудник лаборатории моделирования и анализа экономических процессов; зам. декана экономического факультета

Институт экономики и организации промышленного производства СО РАН; Новосибирский государственный университет пр. Акад. Лаврентьева, 17, г. Новосибирск, 630090, Российская Федерация; ул. Пирогова, 1, г. Новосибирск, 630090, Российская Федеращия

\section{DATA ABOUT THE AUTHORS}

Otmakhova Yuliya Sergeevna, Candidate of Science (Economics), Senior Researcher; Head of Laboratory "Food security research center"

Institute of Economics and Industrial Engineering SB RAS; Novosibirsk State University

17, Academician Lavrentiev Ave., Novosibirsk, 630090, Russian Federation; 1, Pirogov Str., Novosibirsk, 630090, Russian Federation otmakhovajs@yandex.ru

SPIN-code: 4132-9548

ORCID: 0000-0001-8157-0029

Scopus AuthorID: 57194720805

Ibragimov Naimdzon Mulaboevich, Candidate of Science (Economics), Senior Researcher; Vice Dean

Institute of Economics and Industrial Engineering SB RAS; Novosibirsk State University

17, Academician Lavrentiev Ave., Novosibirsk, 630090, Russian Federation; 1, Pirogov Str., Novosibirsk, 630090, Russian Federation naimdjon.ibragimov@gmail.com

SPIN-code: 6608-4495

Scopus AuthorID: 57202757558 